\title{
Experiência de cárie em crianças de 3 a 5 anos de idade em escolas públicas do município de Porto Velho-RO
}

Caries experience in 3-5 year-old children in public schools in the city of Porto Velho-RO

Experiencia de caries en niños de 3 a 5 años de edad en escuelas públicas del municipio de Porto Velho-RO

\author{
Ana Giselle Aguiar DIAS ${ }^{1,2}$ \\ Alberto Carlos Botazzo DELBEM ${ }^{2}$ \\ Caio SAMPAIO ${ }^{2}$ \\ Egídio Bidô da SILVA NETO \\ Gerson Soares RIBEIRO ${ }^{1}$ \\ Juliano Pelim PESSAN ${ }^{2}$ \\ ${ }^{I}$ Centro Universitário São Lucas, 76805-846 Porto Velho-RO, Brasil \\ ${ }^{2}$ Universidade Estadual Paulista (UNESP), Faculdade de Odontologia de Araçatuba, 16015-050 Araçatuba- SP, Brasil
}

\section{Resumo}

O objetivo deste estudo foi avaliar a experiência de cárie em crianças de 3-5 anos de idade, em escolas públicas de Porto Velho-RO, através dos índices epidemiológicos preconizados pela OMS (ceo-s e ceo-d). Crianças $(n=280)$ foram examinadas após escovação supervisionada, utilizando espátulas de madeira e sob iluminação artificial. O coeficiente kappa obtido (concordância interexaminador) foi de 0,95. Os índices ceo-s e ceo-d médios obtidos foram de 4,75 e 2,77, respectivamente, considerando todas as crianças, observando um aumento nestes índices de acordo com a idade. Em relação ao gênero, os índices correspondentes foram 5,01 e 2,71 para meninos e 4,54 e 2,82 para meninas. O componente "cariado" foi responsável por 98 e $90 \%$ dos índices ceo-s e ceo-d, respectivamente. Verificou-se alta incidência de cárie, sugerindo a necessidade de direcionar a esta população uma abordagem preventiva e curativa mais abrangente, com políticas de prevenção mais eficientes, atendendo às necessidades da população estudada.

Descritores: Cárie Dentária; Saúde Bucal; Criança; Índice CPO; Inquéritos Epidemiológicos.

\section{Abstract}

The aim of this study was to evaluate the caries experience in children aged 3 to 5 years old, from public schools in the city of Porto VelhoRO, using the epidemiological indexes recommended by WHO (dmf-s and dmf-t). Children $(n=280)$ were examined after supervised brushing, using wooden spatulas and under artificial lighting. The kappa index obtained (inter-examiner agreement) was 0.95 . Mean dmf-s and dmf-t indexes were 4.75 and 2.77, respectively, considering all children, with an increase in these values according to age. Regarding gender, the corresponding indexes were 5.01 and 2.71 for boys, and 4.54 and 2.82 for girls. The "carious" component accounted for 98 and $90 \%$ of the dmf-s and dmf-t indexes, respectively. A high incidence of dental caries was observed in the present study, suggesting the need to target more careful and comprehensive preventive and curative approaches, with more efficient preventive policies that meet the needs of the population studied.

Descriptors: Dental Caries; Oral Health; Child; DMF Index; Epidemiological Surveys.

\section{Resumen}

El objetivo de este estudio fue evaluar la experiencia de caries en niños (3-5 años), en escuelas públicas de Porto Velho-RO, por los índices epidemiológicos preconizados por la OMS (ceo-s y ceo-d). Niños $(n=280)$ fueron examinados después de cepillado supervisado, utilizando espátulas de madera y bajo iluminación artificial. El coeficiente kappa obtenido (concordancia interexaminador) fue de 0,95. Los índices ceos y ceo-d medios obtenidos fueron de 4,75 y 2,77 , respectivamente, considerando todos los niños, observando un aumento en estos índices de acuerdo con la edad. En relación al género, los índices correspondientes fueron 5,01 y 2,71 para niños y 4,54 y 2,82 para niñas. El componente "cariado" fue responsable de 98 y 90\% de los índices ceo-s y ceo-d, respectivamente. Se verificó alta incidencia de caries, sugiriendo la necesidad de un abordaje preventivo y curativo más amplio, con políticas de prevención más eficientes, atendiendo a las necesidades de la población estudiada.

Descriptores: Caries Dental; Salud Bucal; Niño; Índice CPO; Encuestas Epidemiológicas.

\section{INTRODUÇÃO}

O empenho na redução da incidência da cárie dentária vem fazendo com que cada vez mais a abordagem em relação ao seu controle seja discutida. Atualmente, define-se cárie dentária como uma doença dieta-dependente, sendo o açúcar o fator determinante para o seu estabelecimento. Apesar da necessidade do acúmulo de biofilme na superfície dentária, este não é o fator primordial para o seu desenvolvimento, uma vez que é indispensável a presença de açúcar sobre a produção dos ácidos envolvidos na dinâmica cariogênica; portanto, o microorganismo empenha um papel participativo no processo cariogênico, e não determinante, o que nos propõe que a cárie não seja uma doença infecciosa e transmissível $^{1,2}$. Em acréscimo, a literatura ainda nos mostra forte evidência sobre a associação entre o seu estabelecimento com aspectos comportamentais ${ }^{3}$, interações biológicas ${ }^{6}$, e condições econômicosociodemográficas $^{5-7}$, o que explica as dificuldades no controle da incidência da doença ainda nos dias atuais.

A cárie dentária é uma das mais prevalentes afecções bucais, afetando aproximadamente $90 \%$ da população mundial ${ }^{8}$. Atualmente, cerca de $46 \%$ das crianças brasileiras aos cinco anos de idade estão livres deste problema ${ }^{9}$, embora a incidência de doenças da cavidade bucal varia de uma população para outra, sendo considerados fatores causais condições nutricionais, hábitos relacionados ao estilo de vida, localização geográfica, além da situação socioeconômica do indivíduo ${ }^{10}$. Apesar de disponibilizados pelo governo, os programas de prevenção em saúde bucal não atingem efetivamente a população mais carente, que, sem acesso às informações sobre tratamentos, ou recursos para este fim, acabam procurando por tratamento odontológico apenas quando o quadro de dor já se encontra instalado $^{11}$. 
Estudos recentes constataram uma elevada incidência de comprometimento de dentes decíduos por etiologia cariosa ${ }^{12-14}$. Cardoso et al. ${ }^{13}$ avaliaram 404 fichas de atendimentos assistidos em uma faculdade de odontologia do Rio Grande do Sul, constatando que $42,6 \%$ da amostra apresentou perda precoce de molares decíduos, tendo a cárie dentária como a origem exclusiva destes danos. Resultados compatíveis foram encontrados nos trabalhos de Silva et al. ${ }^{14}$ e Almeida et al. ${ }^{15}$, ambos realizados no estado de Rondônia. Os autores observaram uma grande incidência de cárie em dentes decíduos, sendo o componente cariado o que mais contribuiu para as altas prevalências observadas com uma grande contribuição do componente cariado.

Embora a fluoretação de água seja considerada pela Organização Mundial da Saúde (OMS) uma medida reconhecidamente eficaz na prevenção da cárie dentária ${ }^{16} \mathrm{e}$, segundo a lei $\mathrm{N}^{\circ}$ 6.050 de $24 / 05 / 1974^{17}$ obrigatória para todas as cidades brasileiras que possuam estação de tratamento, o Ministério da Saúde admite uma cobertura restrita à aproximadamente $60 \%$ da população. Tendo em consideração a significativa desigualdade entre as macro regiões do Brasil, a região Norte possui uma das menores taxas de abrangência, apresentando apenas $30 \%$ de abastecimento fluoretado ${ }^{18}$. Narvai et al. ${ }^{19}$ por meio de um estudo comparativo entre os índices CPO-D das capitais com água fluoretada e não fluoretada, constataram uma elevação estatisticamente significativa nas médias de três cidades cujo abastecimento de água não era fluoretado (Belém, Macapá e Porto Velho) sendo que Porto Velho apresentou o maior índice para a faixa etária de 12 anos $(4,15)$ considerando o conjunto de todas as capitais ${ }^{9}$. Considerando a dentição decídua, Porto Velho apresentou o quarto maior índice ceo-d entre as capitais $(2,89)$, sendo inferior apenas em comparação a Rio Branco $(2,98)$, Cuiabá-MT $(3,17)$ e Boa Vista- RR $(3,51)^{9}$.

Considerando a alta prevalência de cárie na região Norte do país e a escassez de levantamentos epidemiológicos na dentição decídua em Porto Velho, o presente trabalho propôs avaliar a experiência de cárie dentária em crianças de três a cinco anos de idade, provenientes de escolas públicas do município de Porto Velho-RO. Para uma análise mais aprofundada, foram utilizados os índices ceo-s e ceo-d, para permitir que a contribuição de cada componente destes índices (dentes/superfícies cariados, com extração indicada ou obturados) fosse analisada.

\section{MATERIAL E MÉTODO}

\section{- Seleção dos voluntários}

O presente estudo foi aprovado pelo Comitê de Ética de Pesquisa em Seres Humanos da
Faculdade de Odontologia de Araçatuba FOAUNESP (CAAE 36332114.8.0000.5420). O Termo de Consentimento Livre e Esclarecido (TCLE) foi devidamente assinado pelos pais ou responsáveis legais, os quais foram orientados quanto ao objetivo da pesquisa, sobre o respeito pela dignidade humana e pela total proteção dos envolvidos na pesquisa, conforme a resolução $\mathrm{n}^{\circ}$ 466/12. O Termo de Assentimento do Menor (TAM) também foi obtido, concordando em participar do referido estudo.

A amostra da pesquisa foi composta por duzentos e oitenta crianças, entre 3 e 5 cinco anos de idade, que frequentavam doze escolas públicas do município de Porto Velho-RO, sendo a população total destas composta por quinhentos alunos. Os voluntários não eram consumidores de água fluoretada proveniente de abastecimento público.

Os critérios de exclusão foram: não obtenção do TCLE e do TAM; crianças portadoras de necessidades especiais ou cujo comportamento inviabilizasse a realização do exame; crianças fora da faixa etária proposta pelo estudo; ou voluntários que faltaram no dia do exame.

\section{- Coleta de Dados}

A coleta de dados foi realizada por dez examinadores e dez anotadores, utilizando os equipamentos de proteção individual (EPI). A análise foi executada dentro do horário escolar, respeitando o intervalo do lanche, tanto no período matutino quanto no vespertino.

Antes da análise da cavidade bucal, os examinadores escovaram os dentes das crianças com escovas dentais e dentifrício fluoretado. Em seguida, as crianças foram encaminhadas para uma sala cedida pela coordenação da escola, nas quais os examinadores se sentavam de frente para as crianças para a realização do exame clínico, o qual foi realizado com auxílio de lanterna de cabeça e espátulas de madeira descartáveis.

Um odontograma foi elaborado para quantificar a experiência de cárie por superfície do dente, adotando-se as seguintes condições (códigos): hígido (A), cariado (B), obturado com cárie (C), obturado sem cárie (D), com extração indicada (E), com selante de fóssulas e fissuras $(\mathrm{G})$ e outros $(\mathrm{O})$. Esta última condição compreendia dentes traumatizados, esfoliados ou com outra condição não relevante para o presente estudo.

- Calibração

A fim de se buscar precisão no exames, minimizando erros diagnósticos, foi adotado a calibração segundo os critérios padronizados pela Organização Mundial da Saúde (OMS) ${ }^{20}$.

$\mathrm{O}$ treinamento teórico foi realizado em forma de aula expositiva e dialogada, abordando os critérios de diagnóstico através da demonstração de diferentes condições de saúde bucal de crianças por projeção de imagens, além da revisão das formas de registro e 
códigos. O treinamento prático seguiu a forma de exame bucal, para assegurar que cada examinador pudesse examinar dentro de um padrão consistente. Dez examinadores realizaram os exames em diversas crianças com o auxílio de dez anotadores. Para tanto se buscou a calibração interexaminador (concordância interexaminador).

A calibração interexaminador ocorreu de modo que respeitasse os critérios de Frias $^{21}$ em relação à porcentagem geral de concordância (PGC) entre os observadores, sendo realizada a repetição dos exames em pelo menos $10 \%$ da amostra e comparando os resultados. Caso o resultado fosse insatisfatório, os examinadores deveriam se reunir e discutir as diferenças encontradas entre os diagnósticos. Se um ou mais examinadores continuassem a registrar mais ou menos itens que o restante do grupo, e as tentativas de correção falhassem, estes deveriam ser excluídos do levantamento. Foi obtido o índice de Kappa de 0,95 durante a calibração, o que é considerado excelente ${ }^{22}$.

\section{- Análise estatística}

Os dados foram tabulados em planilha (Microsoft Excel) e submetido a análise estatística descritiva, por meio de valores absolutos e relativos. Em acréscimo, os dados foram analisados pelo teste $\mathrm{t}$ não pareado (comparação entre gêneros) e teste de Kruskal Wallis, seguido pelo teste de Dunn (comparação entre as idades), utilizando o programa SigmaPlot, versão 12.0. O nível de significância adotado foi de $5 \%$.

\section{RESULTADOS}

Duzentos e oitenta pré-escolares entre 3 e 5 anos de idade foram examinados, obtendo-se uma taxa de resposta de $56 \%$ em relação ao total de crianças que frequentavam as escolas $(n=500)$. Do total de crianças examinadas, 164 apresentavam histórico da doença, correspondendo a uma prevalência de aproximadamente $59 \%$ de cárie dentária nas crianças examinadas. A distribuição das crianças de acordo com o índice ceo-s é apresentada na Figura 1.



Figura 1: Distribuição das crianças examinadas de acordo com o índice ceo-s $(n=280)$.
Houve uma maior participação de meninas ( $60 \%)$ e de crianças de 4 anos de idade ( $56 \%)$ na população estudada, conforme descrito na Tabela 1 . A prevalência de cárie em meninas foi $10 \%$ menor que em meninos utilizando o índice ceo-s, e $4 \%$ maior em meninos utilizando o índice ceo-d, embora sem diferença significativa em comparação ao gênero feminino $(p=0,907$ e $p=0,663$, respectivamente para ceo-s e ceo-d). Observou-se um aumento na prevalência da doença de acordo com a idade, havendo diferença significativa apenas para as idades de 3 e 4 anos utilizando o índice ceo-d. Considerando todas as superfícies avaliadas, a maioria $(\sim 95 \%)$ recebeu o escore "hígido" (Tabela 2). Com relação ao índice ceo-s, o componente cariado foi responsável por $\sim 92 \%$ do valor obtido (Tabela 3 ).

Tabela 1. Distribuição das crianças avaliadas no estudo de acordo com gênero, idade e índices ceo-s (superfícies cariadas, indicadas para extração e obturadas) e ceo-d (dentes cariados, indicados para extração e obturados)

$\left.\begin{array}{cccccc}\hline \multirow{2}{*}{\text { Gênero }} & & \boldsymbol{n} & \mathbf{\%} & \text { ceo-s (DP) } & \text { ceo-d (DP) } \\ & \text { Masculino } & 113 & 40,4 & 5,01(7,77) & 2,71(3,37) \\ & \text { Feminino } & 167 & 59,6 & 4,54(6,97) & 2,82(3,20) \\ \hline \multirow{2}{*}{\text { Idade }} & 3 \text { anos } & 70 & 25,0 & 3,47(5,99) & 1,94(2,95) \\ & 4 \text { anos } & 158 & 56,4 & 4,94(7,42) & 2,95(3,19)\end{array}\right] *$

$n=$ número de crianças avaliadas; $\%=$ porcentagem. Dados de ceo-s e ceo-d apresentados como média (desvio padrão).

* Indica diferença significativa entre as médias (teste de Dunn, $\mathrm{p}<0,05$ ).

Tabela 2. Condição das superfícies avaliadas (número absoluto e relativo)

\begin{tabular}{ccc}
\hline Superfícies (código) & $\boldsymbol{n}$ & $\%$ \\
\hline Hígidas (A) & 26.385 & 94,23 \\
Cariadas (B) & 1.259 & 4,50 \\
Obturado com cárie (C) & 35 & 0,13 \\
Obturado sem cárie (D) & 45 & 0,16 \\
Extração Indicada (E) & 70 & 0,25 \\
Selante (G) & 6 & 0,02 \\
Outro (O) & 200 & 0,71 \\
\hline Total & 28.000 & 100,00 \\
\hline
\end{tabular}

Tabela 3. Índices ceo-s (superfícies cariadas, indicadas para extração e obturadas) e ceo-d (dentes cariados, indicados para extração e obturados) considerando todas as crianças avaliadas

\begin{tabular}{ccc}
\hline $\begin{array}{c}\text { Componentes dos índices ceo-s } \\
\text { e ceo-d }\end{array}$ & ceo-s (DP) & ceo-d (DP) \\
\hline Cariados & $4,65(7,23)$ & $2,50(3,04)$ \\
Indicados para extração & $0,03(0,27)$ & $0,05(0,40)$ \\
Obturados & $0,07(0,51)$ & $0,21(0,80)$ \\
\hline Total & $\mathbf{4 , 7 5}(7,32)$ & $\mathbf{2 , 7 7 ( 3 , 2 6 )}$ \\
\hline
\end{tabular}

Média (DP), $n=280$.

\section{DISCUSSÃO}

Embora a caracterização da situação da cárie dentária de uma população seja realizada principalmente na faixa etária de $12 \operatorname{anos}^{23}$, a análise de incidência meramente no período pós-eruptivo nos restringiria somente aos cuidados curativos, inviabilizando maiores retornos em relação à abordagem preventiva. Portanto, é indispensável que 
seja conferido às fases eruptivas dos dentes um cuidado apropriado, visando a garantir a importância da atenção bucal precoce sobre a prevenção das afecções bucais - em especial - a cárie dentária; sobre o favorecimento da instalação de hábitos preventivos individuais desejáveis ${ }^{24,25}$; além de ser economicamente vantajoso ${ }^{25}$. Ademais, do ponto de vista epidemiológico, é favorável para as estratégias de promoção de saúde pública que a cárie seja diagnosticada ainda na sua fase inicial, viabilizando o emprego de ações para o controle e reversão do quadro, impedindo que a doença atinja estágios irreversíveis ${ }^{11}$.

No presente estudo, observou-se um número elevado de superfícies cariadas em relação às superfícies restauradas (com ou sem cárie), corroborando com levantamentos prévios realizados em cidades do interior do Estado de São Paulo em crianças na mesma faixa etária, nos quais a proporção do elemento "cariado" variou entre 78,3 e $87,9 \%{ }^{26,27}$. Tais resultados sugerem que até mesmo as abordagens curativas podem estar sendo ineficientes e pouco abrangentes nestas regiões, visto que o tratamento das lesões instaladas (visando a minimizar os danos da cárie dentária) não está ocorrendo. Este cenário potencialmente favorece o desenvolvimento destas lesões para estágios mais avançados, com maiores consequências para o paciente.

Pôde-se observar, também, índices ceo-d bem elevados, visto que para as crianças de 5 anos de idade, o presente estudo encontrou média de 3,13, dados superiores ao apontado pelo SBBRASIL $^{9}$, que apresentava para a mesma faixa etária 2,89 na cidade de Porto Velho. Ademais, contribuindo com os achados, comprova-se a alta prevalência de cárie dentária nesta população, considerando que mais da metade das crianças incluídas na pesquisa apresentavam ao menos um dente decíduo com experiência de cárie. Um aspecto importante foi a observação de um aumento no índice ceo-s médio de acordo com a idade das crianças $(3,41,5,42$ e 6,13, respectivamente, para crianças de 3,4 e 5 anos de idade), confirmando a natureza progressiva da doença cárie. Índices ceo-s ainda maiores foram obtidos em crianças entre 6 e 10 anos de idade (ceo-s variando entre 9,70 e 10,95$)^{28}$, enfatizando a necessidade da implementação de medidas preventivas, educativas e curativas eficazes, a fim de se controlar a doença nas dentaduras decídua e mista, visando a uma melhor condição de saúde bucal na dentição permanente.

Diferentemente do presente levantamento, o qual observou índice ceo-s de 5,70 para crianças de 5 anos de idade, valores muito menores foram observados nas cidades de Piracicaba - SP $(3,08$, aos 5 anos de idade $)^{5}$ e Bauru - SP (1,59, 2,23 e 2,38, respectivamente para 3,4 e 5 anos de idade $)^{26}$. A discrepância entre os dados do presente estudo e os dos estudos supracitados pode ser justificada pela ausência de abastecimento de água fluoretada na cidade de Porto Velho-RO, bem como por possíveis diferenças socioeconômicas, políticas e culturais. Neste sentido, vários estudos salientam a relação entre o estabelecimento e aumento de incidência da cárie com os aspectos demográfico-socioculturais e com o acesso da população a recursos e programas de manutenção de saúde bucal. Smith et $\mathrm{al.}^{29}$, constataram grande discrepância nos índices de cárie em crianças de 4-5 anos de idade, residentes em áreas rurais, remotas e metropolitanas da Austrália, tendo encontrado valores de ceo-s, respectivamente, de 4,2, 8,0 e 1,9, e ceo-d, respectivamente de 1,5, 3,5 e 1.1. Estes achados evidenciam a necessidade de programas de promoção de saúde bucal que tenham maior alcance a todos os estratos populacionais. Corroborando com estes achados, uma alta incidência da doença foi observada em uma região ribeirinha no Estado de Rondônia, na Região Amazônica, sendo estes dados atribuídos à falta de acesso aos cuidados em saúde bucal, consumo de água não fluoretada e à necessidade de educação em saúde bucal ${ }^{14}$. Em acréscimo, os resultados do SBBRASIL ${ }^{9}$ evidenciam maiores índices ceo-d nas regiões Norte e Nordeste do país, sendo mais prevalente a cárie não tratada em crianças da pele cor preta e parda, e em crianças com renda familiar menos elevada, corroborando com a importância de se levar em consideração os aspectos sociodemográficos na execução de um programa de saúde bucal mais efetivo e abrangente ${ }^{30}$.

\section{CONCLUSÃO}

Os resultados do presente estudo evidenciam que a prevalência de cárie dentária ainda é alta no município de Porto Velho - RO, considerando a média do país para a faixa etária estudada. O aumento na prevalência da doença de acordo com a idade reforça o caráter progressivo da doença. Assim, considerando que o componente "não tratado" foi o que mais contribuiu para os índices de cárie avaliados, os dados obtidos enfatizam a importância da implementação de programas não somente de caráter preventivo, mas também com abordagem curativa mais eficiente e de maior alcance à população, considerando as possíveis consequências para o paciente decorrentes do não tratamento das lesões cavitadas.

\section{REFERÊNCIAS}

1. Sheiham A, James WPT. Diet and dental caries: the pivotal role of free sugars reemphasized. J Dent Res. 2015;94(10):1341-47.

2. Moynihan PJ, Kelly SA. Effect on caries of restricting sugars intake: systematic review to inform WHO guidelines. J Dent Res. 2014; 93(1):8-18.

3. Fraiz FC, Walter LRF. Study of the factors associated with dental caries in children who 
receive early dental care. Pesqui Odontol Bras. 2001;15(3):201-7.

4. Silva PVD, Troiano JA, Nakamune ACMS, Pessan JP, Antoniali C. Increased activity of the antioxidants systems modulate the oxidative stress in saliva of toddlers with early childhood caries. Arch Oral Biol. 2016;70:62-6.

5. Cortellazzi KL, Tagliaferro EPS, Assaf AV, Tafner APMF, Ambrosano GMB, Bittar TO et al. Influência de variáveis socioeconômicas, clínicas e demográfica na experiência de cárie dentária em pré-escolares de Piracicaba, SP. Rev bras epidemiol. 2009;12(3):490-500.

6. Baldani MH, Narvai PC, Antunes JLF. Cárie dentária e condições sócio-econômicas no Estado do Paraná, Brasil. Cad Saúde Publica. 2002;18(3):755-63.

7. Castilho ARF, Mialhe FL, Barbosa TS, PuppinRontani RM. Influence of family environment on children's oral health: a systematic review. J Pediatr. 2013;89(2):116-23.

8. Duarte JML. Higiene Oral e Prevalência de Cárie Dentária em Crianças e Adolescentes [dissertação]. Porto: Universidade Fernando Pessoa; 2014.

9. BRASIL. Ministério da Saúde. SB Brasil 2010: Pesquisa Nacional de Saúde Bucal Resultados Principais. Brasília, 2011.

10.Tommasi AF. Exame Clínico. Diagnóstico em Patologia Bucal. São Paulo:Pancast; 1989.

11.Figueiredo PBA, Silva ARQ, Silva AI, Silva BQ. Perfil do atendimento odontopediátrico no setor de urgência e emergência da clínica odontológica do Centro Universitário do Pará - CESUPA. Arq Odontol, Belo Horizonte. 2013;49(2):88-95.

12.Losso EM, Tavares MCR, Silva JYB da, Urban CA. Severe early childhood caries: an integral approach. J Pediatr. 2009;85(4):295-300.

13. Cardoso L, Zembruski C, Fernandes DSC, Boff I, Pessin V. Avaliação da prevalência de perdas precoces de molares decíduos. Pesqui bras odontopediatria clín. integr. 2005;5(1):17-22.

14. Silva RHA, Castro RFM, Cunha DCS, Almeida CT, Bastos JRM, Camargo LMA. Cárie dentária em população ribeirinha do Estado de Rondônia, Região Amazônica, Brasil, 2005/2006. Cad Saúde Publica. 2008;24(10):2347-53.

15. Almeida DL, Nascimento DOR, Rocha ND, Dias AGA, Castro RFM, Closs PS. Avaliação da saúde bucal de pré-escolares de 4 a 7 anos de uma creche filantrópica. RGO Rev Gaúcha Odontol. 2011;59(2):271-75.

16.Petersen PE, Lennon MA. Effective use of fluorides for the prevention of dentalcaries in the 21st century: the WHO approach. Community Dent Oral Epidemiol. 2004;32(5):319-21.
17.BRASIL. Lei $\mathrm{N}^{\circ} 6.050$ de 24 de maio de 1974. Diário Oficial da União - Seção 1 - 27/5/1974, Página 6021.

18. Antunes JLF, Narvai PC. Dental health policies in Brazil and their impact on health inequalities. Rev Saude Publica. 2010;44(2):360-65.

19. Narvai PC, Frias AC, Fratucci MVB, Antunes JLF, Carnut L, Frazão P. Fluoretação da água em capitais brasileiras no início do século XXI: a efetividade em questão. Saúde debate. 2014;38(102):562-71.

20.Peres MA, Traebert J, Marcenes W. Calibração de Examinadores para Estudos Epidemiológicos. Cad Saúde Pública. 2001;17(1)153-59.

21.Frias AC. Estudo de Confiabilidade do Levantamento Epidemiológico de Saúde Bucal Estado de São Paulo 1998 [dissertação]. São Paulo: Faculdade de Saúde Pública da USP; 2000.

22.Landis, JR, Koch GG. The measurement of observer agreement for categorical data. Biometrics. 1977;33(1):159-74.

23. Global goals for oral health in the year 2000 . Federation Dentaire Internacionale. Int Dent J. 1982;32(1):74-7.

24. Borges ESM, Toledo OA. A prevalência de carie em crianças de 0-5 anos: avaliação após cinco anos de um programa preventivo. Rev $\mathrm{ABO}$ nac. 1999;7(5):298-303.

25.Walter RLF, Nakama R. Prevenção da cárie dentária através da identificação e controle dos fatores de risco em bebes - Parte I. J Bras Odontop Odontol Bebê. 1998;1(3):91-100

26. Oliveira TM, Silva TC, Sakai VT, Prestes MP, Honorio HM, Magalhães AC et al. Comparação entre os índices ceos e ceos modificado em bebês e pré-escolares. Rev Odontol da Univ Cid São Paulo. 2008;20(2):128-33.

27. Amaral RC, Hoffmann RHS, Cypriano S, Sousa MLR, Silva AAZ. Prevalência de cárie e necessidades de tratamento em pré-escolares e escolares de Rafard - SP -Brasil. Cienc Odontol Bras. 2006;9(3):87-93.

28. Moura MS, Santos-Pinto LAN, Giro EMA, Cordeiro RCL. Cárie dentária relacionada ao nível socioeconômico em escolares de Araraquara. Rev Odontol UNESP. 1996;25(1):97-107.

29. Smith L, Blinkhorn A, Moir R, Brown N, Blinkhorn F. An assessment of dental caries among young Aboriginal children in New South Wales, Australia: a cross-sectional study. BMC Public Health. 2015;15:1314.

30.Ardenghi TM, Piovesan C, Antunes JLF. Desigualdades na prevalência de cárie dentária não tratada em crianças pré-escolares no Brasil. Rev Saúde Pública. 2013;47(Suppl 3):129-37. 


\section{CONFLITO DE INTERESSES}

Os autores declaram não haver conflitos de interesse.

\section{AUTOR PARA CORRESPONDENCIA}

\section{Juliano Pelim Pessan}

juliano.pessan@unesp.br

Submetido em 12/12/2018

Aceito em 12/03/2019 